
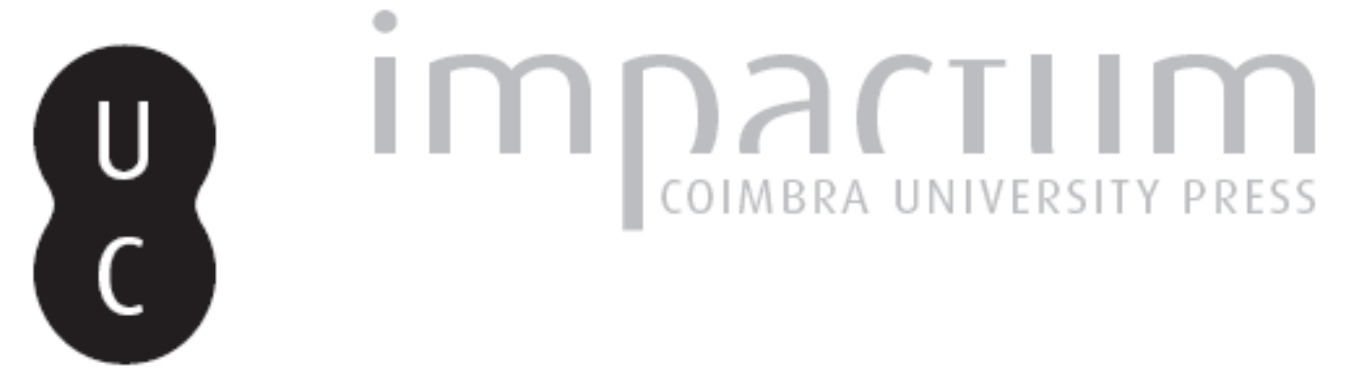

\title{
L'épigraphie du nord du Portugal: bilan et perspectives
}

\author{
Autor(es): $\quad$ Roux, P. le; Tranoy, A.
}

Publicado por: Imprensa da Universidade de Coimbra

URL persistente:

URI:http://hdl.handle.net/10316.2/45672

DOI:

DOI:https://dx.doi.org/10.14195/1647-8657_23_2

Accessed : $\quad$ 26-Apr-2023 11:53:07

A navegação consulta e descarregamento dos títulos inseridos nas Bibliotecas Digitais UC Digitalis, UC Pombalina e UC Impactum, pressupõem a aceitação plena e sem reservas dos Termos e Condições de Uso destas Bibliotecas Digitais, disponíveis em https://digitalis.uc.pt/pt-pt/termos.

Conforme exposto nos referidos Termos e Condições de Uso, o descarregamento de títulos de acesso restrito requer uma licença válida de autorização devendo o utilizador aceder ao(s) documento(s) a partir de um endereço de IP da instituição detentora da supramencionada licença.

Ao utilizador é apenas permitido o descarregamento para uso pessoal, pelo que o emprego do(s) título(s) descarregado(s) para outro fim, designadamente comercial, carece de autorização do respetivo autor ou editor da obra.

Na medida em que todas as obras da UC Digitalis se encontram protegidas pelo Código do Direito de Autor e Direitos Conexos e demais legislação aplicável, toda a cópia, parcial ou total, deste documento, nos casos em que é legalmente admitida, deverá conter ou fazer-se acompanhar por este aviso.

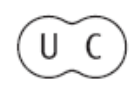


FACULDADE DE LETRAS

INSTITUTO DE ARQUEOLOGIA

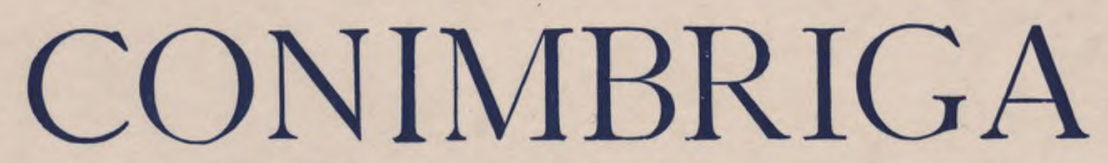

VOLUMEXXIII

UNIVERSIDADE DE COIMBRA

1984 
P. LE ROUX

Professeur à PUniversité de Toulouse-Le Mireil

A. Tranoy

Professeur à PUniversité de Poitiers

L'ÉPIGRAPHIE DU NORD DU PORTUGAL:

BILAN ET PERSPECTIVES

Conimbriga, XXIII (1984) p. 19-41

RÉSUMÉ : Après un rappel historique et géographique, est presenté un tableau de Pépigraphie du Nord du Portugal à partir d'un bilan numérique. Les difficultés d'étude et les problèmes de publication restent une préoccupation dominante. L'exemple du Trás-os-Montes sert à illustrer l'évolution de la recherche épigraphique et la richesse des renseignements que l'on peut tirer de cette documentation pour l'étude de la société (onomastique, filiation, décors funéraires).

RESUmo: OS autores fazem um balanço das inscrições romanas que hoje se conhecem no Norte de Portugal, região cujas condições históricas e geográficas recordam de modo geral e sumário. São cerca de 720 inscrições, contra 358 publicadas no CIL II. As dificuldades de ejstudo, em parte devidas à dispersão dos monumentos e, noutra pairie, à natureza do material utilizado (essencialmente granito) são salientados pelos autores. O exemplo da epigrafia transmontana ilustra a evolução dos estudos epigráficos e a riqueza das informações que se podem extrair destes documentos para o estudo da sociedade (onomástica, filiação, decorações funerárias). 
(Página deixada propositadamente em branco) 


\section{L'ÉPIGRAPHIE DU NORD DU PORTUGAL: BILAN ET PERSPECTIVES *}

Depuis quelques années, des enquêtes épigraphiques ont été menées dans le nord du Portugal, soit par des chercheurs portugais, soit par nous-mêmes, présents au Portugal dans le cadre des travaux et des recherches programmés par le Centre Pierre Paris de Bordeaux. Nous avons déjà eu l'occasion d'en exposer quelques résultats lors du Illème Congrès archéologique national de Porto en $1973 \mathrm{i}^{1}$ ). En réalité, grâce à la perspicacité de chercheurs locaux et à la collaboration de nos amis portugais que nous tenons à remercier pour leur fructueuse participation dans nos prospections (2), la collection épigraphique du Nord du Portugal a pu s'enrichir de nombreux documents nouveaux et importants, en même temps qu'étaient révisées les inscriptions déjà connues, mais qui présentaient des difficultés ou des erreurs de lecture. Diverses publications ont rendu compte d'une partie de ces travaux $\left({ }^{3}\right)$. Mais il nous a paru opportun de mettre à

* Communication présentée au IY Congresso Nacional de Arqueologia (Faro 1980).

(M P. Le Roux et A. Tranoy, Contribution à Vétude des régions rurales du N.O. hispanique au Haut-Empire: deux inscriptions de Penafiel, III CNA (Porto, 1973), Porto, 1974, p. 249-258; P. LE Roux, Les stèles funéraires de Braga. Remarques sur une nouvelle inscription en vers, «Archeologica Opuscula», I, 1974, p. 41-48.

${ }^{2}$ ) Nous remercions tout particulièrement C. M. Faya de Santarém, C. A. Ferreira de Almeida, Luciano Afonso dos Santos, A. M. Mourinho, J. Parente, J. J. Rigaud de Sousa, J. R. dos Santos Júnior, E. Pires de Oliveira et tous les conservateurs des musées du nord, sans oublier toutes les personnes qui nous ont accueillis dans leur maison au cours de nos prospections et qui nous ont permis d'apprécier l'hospitalité portugaise.

(3) En particulier les communications présentées par J. d'E NCARNAç Âo et A. Tranoy au Seminário de Arqueologia do Noroeste Peninsular, à Guimarães, en juin 1979: O progresso da Epigrafia Romana do NW Peninsular (1970-78) («Actas»... Ill Guimarães 1980 p. 37-41) et Religion et Société à Bracara Augusta (Braga) au Haut-Empire Romain («Actas...», Ill, 1980, p. 67-84).

Conimbriga, 23 (1984), 19-41 
profit le Congrès de Faro pour essayer de dresser un premier bilan des progrès de l'épigraphie du Nord du Portugal. Ce bilan ne pourra être que provisoire, étant donné la richesse épigraphique de cette région et le travail inlassable des chercheurs locaux; cependant il devrait permettre de dégager les caractéristiques et l'originalité du Nord du Portugal, autant par une étude d'ensemble que par un exemple régional, la province du Trás-os-Montes.

\section{Les conditions géographiques et historiques}

Comme la Galice espagnole et une grande partie du Portugal central, les régions comprises entre le Douro, au Sud, et la frontière espagnole, à l'Est et au Nord, appartiennent au prolongement occidental du vieux socle de la Meseta. Mais le relief y est plus heurté et les montagnes y alternent avec les plateaux élevés, disséqués par d'importants réseaux de failles. L'orientation dominante des principaux axes de relief est nord-sud et joue un rôle déterminant pour les divisions régionales de ce secteur.

C'est ainsi que le partage provincial du Nord se fait en fonction des grands massifs qui coupent le pays en deux, depuis les serras de Gérés et de Larouco au Nord, jusqu'au Douro au Sud, avec les serras de Barroso, Cabreira, Padrela, Alvão etMarão; à l'Est de ces montagnes, s'étend le Trás-os-Montes aux paysages rudes, et, à l'Ouest, le Minho, plus riche et très peuplé.

Bordée par une côte plate et rectiligne, la partie occidentale est caractérisée par une multitude de vallées encastrées entre les massifs granitiques et s'écoulant vers la côte: Minho, Lima, Cávado, Ave. Mais pour le reste du pays, le réseau hydrographique dépend du Douro qui constitue l'axe essentiel vers lequel se dirigent les fleuves les plus importants du Nord: Tâmega, Tua, Sabor. En réalité, la vallée du Douro forme surtout une véritable limite entre le Nord et le Centre du Portugal et n'est franchissable qu'en de rares endroits comme Porto, Régua ou la région de Moncorvo.

Ces conditions géographiques se reflètent dans l'évolution historique antique du Nord du Portugal. Ainsi, la répartition 
des sites de castros $\left({ }^{4}\right)$, aspect essentiel du peuplement préromain de cette région, met en évidence le contraste entre la partie occidentale à peuplement dense et les régions orientales où la densité fut moins importante, situation qui se prolongea après la conquête.

Amorcée par l'expédition de Decimus Iunius Brutus en 138136 a.C., cette conquête ne fut réellement achevée que sous Auguste $\left(^{5}\right)$. D'abord rattaché à la nouvelle province de Lusitanie, le Nord du Portugal fut ensuite attribué à la Citérieure, probablement entre 16 et 13 a.C. ( $\left.{ }^{6}\right)$ : dans cette répartition provinciale, le Douro joua pleinement son rôle puisqu'il constitua la limite entre la Lusitanie et la Citérieure dans ce secteur de la Péninsule.

Enfin, sous les Flaviens, le Nord du Portugal fut inclus dans deux conventus, la plus grande partie étant attribuée au conventus Bracaraugustanus, le secteur oriental allant au conventus Asturum. Cette nouvelle disposition tint compte à la fois des conditions géographiques et de la répartition des principaux peuples, en particulier les Callaeci Bracari et les Astures. C'est en fonction de ce cadre que ce pays reçut les influences romaines dont l'épigraphie est un témoin précieux.

\section{Tableau de Pépigraphie du Nord du Portugal}

L'épigraphie constitue, avec l'archéologie, la source essentielle pour l'histoire antique du Nord-Ouest ibérique. En effet, les sources littéraires sont rares et d'utilisation difficile. Strabon fait une description assez générale du Nord de la Péninsule, où il est impossible de préciser ce qui correspond aux régions que nous étudions $\left({ }^{7}\right)$. En dehors des remarques concernant les Zoelae $\left.{ }^{8}\right)$,

$\left.{ }^{4}\right)$ Les castros sont très denses dans le Minho où se trouvent les plu importants comme Briteiros et Sanfins.

(5) Pour le cadre historique, J. Alarcão, Portugal Romano (Historia Mundi), Lisbonne, 2ème éd., 1974, p. 17 s.

$\left.{ }^{6}\right)$ R. Syme, The Conquest of North-West Spain, Legio VII Gemina, León, 1970 p. 104-107.

(7) Strabon, Géographie, livre III.

(8) Pline, XI, 10.

Conimbriga, 23 (1984), 19-41 
Pline l'Ancien ne fait que de brèves allusions aux régions du Nord et ensuite il faut attendre la fin du IVème siècle pour trouver une source littéraire qui s'intéresse plus directement au Portugal, avec l'évêque Hydace $\left({ }^{9}\right)$. On comprend alors toute l'importance que revêt l'épigraphie dont la richesse permet de compenser en partie l'absence de sources littéraires pour le HautEmpire. C'est là sans doute un trait commun aux régions à dominante rurale et «villageoise» des provinces occidentales de l'Empire.

\section{Une première estimation chiffrée.}

Malgré les limites d'une telle étude, il nous a paru utile de tenter une première estimation chiffrée pour mieux saisir la place réelle des inscriptions dans la documentation. Ces chiffres n'auront donc qu'une valeur indicative, mais leur caractère provisoire est en lui-même la preuve du renouvellement permanent de ce matériel, ce qui ne peut être que stimulant pour la recherche. En outre, l'échantillon est déjà suffisamment important pour que les proportions d'ensemble soient considérées comme assez proches de la réalité.

On dispose actuellement d'environ 720 inscriptions latines pour tout le Nord du Portugal. De ce total, on peut mettre à part quelques 200 bornes milliaires qui constituent une des richesses de l'épigraphie du Nord du Portugal. En effet, peu de régions de la Péninsule ont conservé autant de bornes milliaires, pour une bonne partie encore à proximité de la route antique, comme c'est le cas pour la route de Braga à Túy ou de Braga à Astorga par la serra de Gérés. Aussi est-il possible de reconstituer assez fidèlement le réseau routier antique de cette région, desservie par les routes qui, venant de Mérida et de la Lusitanie, reliaient Braga à Lugo et à Astorga $\left({ }^{10}\right)$.

On peut comparer ces deux premiers chiffres avec ceux du C7L, II où le total, pour le Nord du Portugal, est de 358 inscrip-

(9) Hydace, Chronique, édité par A. Tranoy, Paris, 1974 (Sources Chrétiennes, 218-219).

$\left.{ }^{10}\right)$ Ce sont les routes XVI, XVII, XVIII, XIX et XX de Y Itinerarium Antonini.

Conimbriga, 23 (1984), 19-41 
tions dont font partie 131 miliaires. La documentation épigraphique a donc doublé depuis l'établissement du C7L, IL

Si l'on met à part les milliaires qui forment un groupe particulier, il est possible de répartir les 520 inscriptions restantes en fonction des centres d'intérêt et l'on obtient les pourcentages suivants (les chiffres entre parenthèses renvoient au pourcentage obtenue uniquement par le Corpus):

* Inscriptions funéraires $=50 \%(39 \%)$, dont les $2 / 3$ concernent le Trás-os-Montes.

* Inscriptions votives $=31 \%(35 \%)$; dans le domaine religieux, il faut noter l'importance prise par les inscriptions votives pour des divinités indigènes qui représentent près de la moitié des dédicaces. Si l'on ne considère que la partie occidentale, elles dépassent même les dédicaces à des divinités classiques et orientales: 47 dédicaces pour le culte indigène contre 45 pour les cultes classiques et orientaux et 8 pour des divinités indéterminées.

$*$ Inscriptions honorifiques $=4 \%(5 \%)$ dont les plus célèbres sont les dédicaces du pont de Chaves pour les empereurs flaviens et pour Trajan.

* Inscriptions incertaines $=15 \%(21 \%)$ : ce sont essentiellement des inscriptions perdues dont le texte, mal publié, pose des problèmes ou des inscriptions conservées dont la lecture reste très difficile. On peut mettre, pour l'instant, dans ce groupe l'inscription, sur un rocher, de Vilar de Perdizes (Montalegre) dont le texte est loin d'avoir livré tous ses secrets ${ }^{\mathrm{n}}$ ).

Certaines inscriptions échappent à cette classification. C'est le cas de la borne découverte à Alvarelhos (Maia) dont le texte, quoique incomplet, est parfaitement lisible (12) (Fig. 1).

(n) CIL, II, 2476; des solutions de lecture ont été proposées. A. RodRIguez Colmenero, Les Divoi y Devai, receptores de un posible pacto entre dos nuevos grupos gentilicios de la Galicia Romana, «Primera Reunion Gallega de Estudios Clasicos (Santiago-Pontevedra, 2-4 julio 1979)», Saint-Jacques-de-Compostelle, 1981, p. 141-151; X. Pereira Menault et C. A. Ferreira De Almeida, A grande inscrição do Penedo de Remeseiros, Vilar de Perdizes, Montalegre (CIL, II, 2476), «Arqueologia», 4, 1981, p. 142-145.

(12) C. M. Faya de SantaRém, Uma inscrição romana de Alvarelhos. Santo Tirso, «Santo Tirso. Boletim Cultural Concelhio», Vol. I, 1, 1977 lié Série de «O Concelho de Santo Tirso», Boletim Cultural. 
Peut-être avons-nous affaire à une borne-limite entre des groupes dont les Madequisenses seraient l'une des parties concernées?

Enfin, si l'on tient compte d'un classement catégoriel plus détaillé, on peut constater que les inscriptions du Nord du Portugal ne mentionnent que 6 personnages de rang sénatorial, 2 chevaliers et 12 soldats; en revanche, l'onomastique comporte plus de 100 noms de femmes.

\section{Les caractéristiques générales.}

On peut regrouper les caractéristiques générales de l'épigraphie du Nord du Portugal autour de deux points: le matériau utilisé et la dispersion des documents.

Pour la très grande majorité des documents, le matériau utilisé est essentiellement le granit avec toutes les variétés locales que peut présenter cette roche. Aussi se heurte-t-on à toute une série de difficultés, liées à la nature du support. En premier lieu, existe une difficulté de lecture, car certains granits s'effritent et se conservent mal, surtout si la pierre a été exposée aux intempéries. Sur ce point, il serait très souhaitable que les inventeurs ou les chercheurs renoncent définitivement à passer au charbon ou au crayon les traces supposées des lettres sur la pierre: cette méthode est non seulement une source d'erreurs fréquentes, mais elle détériore aussi l'inscription. L'estampage sur le granit étant souvent difficile, il faut utiliser au mieux les techniques d'éclairage artificiel et multiplier les photographies, sans oublier qu'une photographie peut aussi provoquer de mauvaises interprétations et que la lecture directe sur le document reste indispensable.

D'autre part, le lapicide ne peut pas toujours graver le texte aussi régulièrement sur du granit, qu'il le ferait sur du calcaire ou sur du marbre. Cette remarque pose un deuxième problème, la datation du document: dans bien des cas, il vaut mieux renoncer aux critères paléographiques, car des documents mal gravés peuvent très bien être du ler siècle et non du Illème siècle, comme on a souvent tendance à le croire. Inversement, la documentation du IVème siècle reste difficilement identifiable. 
La chronologie est donc un des problèmes fondamentaux posés par les inscriptions du Nord.

L'enquête épigraphique se heurte aussi au problème de la dispersion des documents. Près de $4 / 5$ des inscriptions sont conservées. Il y a, bien sûr, plusieurs musées importants qui les ont regroupées, comme les muséeb archéologiques de Belém, Porto, Chaves, Braga, Bragança et Guimarães, ce dernier ayant une très belle collection épigraphique. Mais beaucoup de pierres sont réparties sur l'ensemble des régions septentrionales, soit dans de petits musées locaux comme à Póvoa de Varzim, Vila Flor ou Miranda do Douro, soit déposées à la Câmara Municipal comme à Montalegre, soit conservées in situ comme dans le village de Saldanha (Mogadouro) ou encore dans une église, réutilisées, comme le fait est fréquent, en bénitier ou en support d'autel ou enfin chez un particulier qui a pu transporter la pierre loin de son lieu de découverte. Le chercheur doit souvent se livrer à une véritable enquête pour retrouver la trace du document. Cette dispersion pose donc le problème de l'accès au document et de sa conservation. En réalité, cette situation est aussi le reflet de l'aspect essentiellement rural de l'épigraphie du Nord du Portugal. En dehors de Braga et de Chaves, la majeure partie de la documentation provient de régions rurales, ce qui donne un intérêt supplémentaire à cette épigraphie.

En effet, elle est le moyen privilégié pour essayer de connaître l'organisation sociale des peuples du Nord et des aspects de leur civilisation, en particulier dans le domaine religieux. C'est par l'épigraphie que l'on peut constater le double phénomène du maintien des cultes indigènes et de la diffusion des cultes classiques. Mais pour obtenir ces résultats, il est indispensable que ces documents soient connus; se pose alors le problème de leur publication.

\section{La publication.}

Avant tout, il faut partir de la situation actuelle. Le Corpus (CIL, II) et VEphemeris Epigraphica (EE, VIII et IX) restent la base de toutes les publications épigraphiques. Mais 
le Corpus pose de nombreux problèmes de classement, de localisation et même de confusion dans la définition des documents.

En effet, lors de l'établissement du CIL, II, E. Hübner se trouva confronté à la multiplication des sites du Nord du Portugal et à l'absence de véritables centres de regroupement en dehors de villes comme Braga ou Chaves. Pour résoudre en partie cette question, il a privilégié quelques villes comme Caldas de Vizela et Bragança et, surtout, il a inséré, sous le titre d'Oppida varia, de nombreux sites allant depuis Porto jusqu'au cours moyen du Douro, le fleuve servant d'axe principal, sans que ce classement tienne compte des importantes différences régionales le long de cette vallée.

La diversité des localisations des inscriptions a aussi provoqué quelques erreurs ou reclassements arbitraires. Ainsi, il n'est pas logique d'inclure dans les inscriptions du Nord du Portugal les pierres provenant de Cárquere (Resende), situé au Sud du Douro, dans le district de Viseu (13). E. Hübner, conscient de cette difficulté, se justifie en expliquant qu'il n'a pas voulu créer de nouveaux addenda $\left({ }^{14}\right)$; mais cette classification ne peut qu'être source de confusion.

Plusieurs inscriptions ont été mal localisées ou publiées deux fois: ainsi, l'inscription de Pombeiro (Beira Alta) a été rangée dans les Oppida varia $\left\{{ }^{15}\right)$ tandis que les autels votifs dédiés aux Nymphes, à Caldeias, étaient enregistrés sous deux rubriques $\left({ }^{16}\right)$. Ces erreurs se reflètent sur la carte pour l'emplacement de Vilariça, localité au Nord-Ouest de Moncorvo et à l'ouest du Sabor, mais largement située par E. Hübner et $\mathrm{H}$. Kiepert à l'est du Sabor, à une trentaine de kilomètres de Moncorvo. Cependant, l'erreur la plus importante est celle qui concerne Castro de Avelãs, situé par E. Hübner à environ $50 \mathrm{kms}$. au Sud-Est de Bragança $\left({ }^{97}\right)$, alors que ce village est à $7 \mathrm{kms}$. à l'Ouest de cette ville: cette mauvaise localisation eut des répercussions sur les limites de conventus $\left({ }^{18}\right)$.

(M) CIL, II, 5570-1, 5573-5580.

(14) CIL, II, p. 818 et 893.

(15) CIL, II, 2380.

(16) CIL, II, 2457 et 5572.

(17) Confusion probable avec Avelanoso.

(18) Infra, pour les limites orientales du conventus Bracaraugustanus. 
Enfin, des inscriptions honorifiques ont été confondues avec des bornes milliaires: si la correction avait été faite pour la dédicace de Caldas das Taipas $\left({ }^{19}\right)$, en revanche, l'inscription de Gostei (Bragança) et la dédicace de Yale de Antas (Chaves) sont restées classées parmi les milliaires alors qu'il s'agit de plaques honorifiques $\left({ }^{20}\right)$.

Depuis l'édition du Corpus et des E. E., les publications locales se sont multipliées, accompagnées de quelques travaux de synthèse. On peut citer, à titre d'exemple, les publications des inscriptions de Roncal (Moncorvo) ( $\left.{ }^{21}\right)$ ou les travaux consacrés à Braga et à sa région (22). Mais, dans le même temps, des inscriptions nouvelles étaient découvertes et restent encore inédites : région de Braga, de Miranda do Douro, de Bragança, etc.... Aussi, devant l'augmentation du nombre des inscriptions, une double nécessité se fait jour. En premier lieu, il nous paraît indispensable d'entreprendre une publication intégrant les documents déjà répertoriés et les nouvelles inscriptions. Cette publication devra tenir compte des données actuelles de la recherche épigraphique, avec une description détaillée du support monumental et du contexte archéologique lorsqu'il existe. D'autre part, il faudra définir un autre type de classement que celui du Corpus et se fonder sur les divisions administratives actuelles, provinces, districts et concelhos, qui tiennent mieux compte des nuances régionales. C'est ainsi que l'on peut regrouper dans un même ensemble les inscriptions de la province du Tràs-os-Montes qui peuvent illustrer cette présentation générale de l'épigraphie du Nord du Portugal.

(19) CIL, II, 4796 - 5560.

(20) CIL, II, 6217; A. Tranoy, Le pseudo-milliaire de S. Cláudio (Gostei, Bragança) et les limites orientales du conventus Bracarum, TAE, XXIII 1980, p. 589-594; CIL II, 4787.

(21) A. Vasco Rodrigues et D. de Pinho brandão, Missão de estudo arqueológico na região da Vilariça, Moncorvc, «Studium Generale», IX, 1962, p. 336-351 (= Studium Generale).

(22) P. Le Roux, AUx origines de Braga (Bracara Augusta), «Bracara Augusta», XXIX, 1975, p. 155-159; P. Le Roux et A. Tranoy, Borne et les indigènes dans le nord-ouest de la péninsule ibérique. Problèmes d'épigraphie et d'histoire, MGV, IX, 1973, p. 127-231 (= Le Roux-Tranoy).

Conimbriga, 23 (1984), 19-41 


\section{IL L’épigraphie du Trás-os-Montes}

\section{Les divisions régionales et le peuplement (Carte I)}

La province du Trás-os-Montes et de l'Alto-Douro est une des régions les plus originales du Portugal, où les coutumes et les traditions locales sont encore très enracinées dans la population malgré les changements progressifs dus à l'émigration. Contrairement au secteur occidental, le Trás-os-Montes est fortement marqué par son caractère continental, accentué par les hautes serras qui le séparent du Minho, comme les serras de Marão et de Alvão. Dans cette région du Nord-Est du Portugal, alternent les plateaux élevés, les serras qui coupent le pays selon une direction $\mathrm{NE} / \mathrm{SW}$, comme les serras de Nogueira, Bornes ou Mogadouro, les bassins d'effondrement, comme le bassin de Vila Flor ou celui de Mirandela. C'est aussi cette même direction que suivent les principaux fleuves, Tâmega, Tua, Rabaçal et Sabor. La variété des paysages est aussi le reflet de la complexité géologique du Trás-os-Montes, où le granit côtoie les schistes comme ceux qui constituent la base des sols de la région viticole de Peso da Régua et de la vallée du Douro, ou encore les marbres, comme ceux du concelho de Vimioso avec les gisements de Santo Adrião. Aussi, retrouve-t-on cette variété dans les matériaux utilisés pour les inscriptions du Trás-os-Montes où, si le granit reste dominant, furent employés, parfois abondamment, les schistes et surtout le marbre, dans la région de Miranda do Douro.

Actuellement, selon les divisions administratives, on distingue deux districts: le district de Vila Real avec 14 concelhos et le district de Bragança avec 12 concelhos. Mais cette division moderne ne correspond pas à la division antique, où cette région se répartissait entre le conventus Bracaraugustanus et le conventus Asturum. La limite orientale entre ces deux conventus a longtemps dépendu de la carte dressée par E. Hübner et H. Kiepert dans le Corpus; or son tracé était faussé par la mauvaise localisation du village de Castro de Avelãs. Sans revenir en détail sur cette question que A. Tranoy a examinée dans un article publié en 1980(23), on peut attribuer au peuple asture des Zoelae le secteur à l'Est

(23) Cf. n. 20. 
des serras de Nogueira et de Bornes; au-delà de ces serras qui forment une limite géographique nette commençait, à l'Ouest, le conventus Bracaraugustanus.

En dehors des Zoelae et des Turodi ou Aquiflavienses, sous la forme romaine, qui occupaient la région de Chaves $\left({ }^{24}\right)$, il est malheureusement très difficile de déterminer le nom des peuples antiques qui vécurent dans le Tràs-os-Montes. Cependant, la répartition des inscriptions permet déjà de se faire une idée des zones favorables à l'occupation humaine antique, qui souvent correspondent au peuplement actuel.

En excluant les milliaires, l'épigraphie du Tràs-os-Montes peut être évaluée provisoirement à environ 260 inscriptions dont la répartition typologique est la suivante:

\begin{tabular}{|l|c|c|c|}
\hline \multirow{2}{*}{ Inscriptions } & \multicolumn{2}{|c|}{ Tràs-os-Montes } & \multirow{2}{*}{$\begin{array}{c}\text { Nord du Portugal } \\
\text { Pourcentage }\end{array}$} \\
\cline { 2 - 3 } & Chiffre & Pourcentage & $50 \%$ \\
\hline Funéraires & 184 & $69 \%$ & $31 \%$ \\
\hline Votives & 53 & $\mathbf{2 0} \%$ & $4 \%$ \\
\hline Honorifiques & 5 & $\mathbf{2} \%$ & $15 \%$ \\
\hline Incertaines & 24 & $9 \%$ & \multicolumn{2}{|c}{} \\
\hline
\end{tabular}

Ce tableau met en évidence la part prépondérante des inscriptions funéraires dans l'épigraphie de cette région. Si l'on reporte sur la carte du Tràs-os-Montes (Carte I) l'ensemble des inscriptions ainsi que les 50 milliaires environ découverts dans cette province, on distingue 5 foyers de densité plus forte: les régions de Chaves, Torre de Moncorvo avec la vallée de Vilariça, Tresminas, Miranda do Douro avec une concentration importante à Picote et enfin la région de Bragança. Dans les deux premiers cas, cette répartition correspond à des régions de culture, que ce soit le bassin fluvial du Tâmega ou la vallée de Vilariça; en revanche, Bragança et

(24) A. Tranoy, A propos des "Callaeci» de Pliru. Épigraphie et,peuplement, «Bracara Augusta», XXXI, 1977, p. 225-233. 
Miranda do Douro sont surtout des régions d'élevage. La concentration épigraphique autour de Tresminas s'explique aisément par les activités minières de cette région $\left({ }^{25}\right)$, qui attire même des personnes originaires d'autres conventus comme celui de Clunia (26). De leur côté, les milliaires dessinent l'axe le plus important qui met en relation Chaves et Astorga par Valpaços, Torre de Dona Chama, où le pont romain témoigne du passage de la route antique $\left({ }^{27}\right)$, et la région de Bragança $\left({ }^{28}\right)$.

Sans prétendre faire ici une étude systématique, ces premières remarques peuvent être complétées par l'onomastique, les types de filiation et quelques aspects des coutumes funéraires à travers les monuments épigraphiques.

\section{L'onomastique du Trds-os- Montes (Carte II).}

Cette présentation des données essentielles de l'onomastique porte sur un total de 278 noms que nous avons classés selon trois groups principaux:

\begin{tabular}{|c|c|c|}
\hline & Chiffre & Pourcentage \\
\hline Noms latins seuls & 128 & $46 \%$ \\
\hline $\begin{array}{c}\text { Noms latins avec } \\
\text { noms indigènes }\end{array}$ & 15 & $5,5 \%$ \\
\hline Noms indigènes seuls & 135 & $41,5 \%$ \\
\hline
\end{tabular}

(25) M. Cardozo, A propósito da laora de ouro na província de Trás-os-Mcntes durante a época romana, dans $R G$, LXIV, 1954, p. 113-141; G. A. FerReira de Almeida, Aspectos da mineração romana de ouro em Jales e Tresminas (Trás-os-Montes), «XII CAN (Jaén, 1971), Saragosse», 1973, p. 553-566 .

(26) Six inscriptions témoignent de cette émigration, partiellement étudiée par G. García Merino, Las tierras del NO de la península ibérica, foco de atración para los emigrantes de la Meseta en época romana, «Hisp. Ant.», Ill, 1973, p. 18-23; cf. aussi A. Tranoy, La Galice Romaine, Paris, 1981. p. 246-256.

$\left.{ }^{27}\right)$ A. M. Mourinho, Ponte romana no rio Tuela e sintese das vias e pontes romanas no nordeste transmontano, TAE, XXIII, 1978, p. 279-288.

(28) L. A. BARRADas, Vias romanas das regiões de Chaves e Rragança, RG, LXVI, 1956, p. 159-238. 
La première remarque qui peut être faite est j'importance de l'élément indigène dans l'onomastique locale, puisque $54 \%$ des noms contiennent un nom indigène. D'autre part, on constate aussi la faiblesse des noms mixtes. Sur ce point, la règle générale est la construction du nom avec un gentilice latin, suivi d'un cognomen indigène: Iulia Camalea $\left({ }^{29}\right)$, Valerius Reburrus $\left({ }^{30}\right)$, Calpurnius Reburrinus, soldat recruté pour Y Ala II Flavia ( ${ }^{31}$ ) (Fig. 2) ou encore Aemilius Balaesus (Fig. 3) pour lequel le texte précise son appartenance à une entité ethnique ( ${ }^{32}$ ). Cependant, pour certaines inscriptions, le nom de la personne est d'origine indigène et son cognomen est latinisé: Amma Valeriana( $\left.{ }^{33}\right)$, Attianus Rufus ( $\left.{ }^{34}\right)$, Laucius Rufînus ( ${ }^{35}$ ).

L'onomastique indigène se répartit sur l'ensemble de la province (Carte II). Le nom prédominant est incontestablement Reburrus et ses dérivés, Reburrinus, -a: 19 cas; puis viennent Camalus (6 cas), Allius (5 cas), Boutius (5 cas), Cloutius (5 cas), Trites ou les formes voisines en Tridius ( 5 cas); les autres noms sont encore moins utilisés: Arro, Arcius, Pintamus, Segontius, etc... Dans le cas de noms à structure indigène exclusive, la très grande majorité des noms sont uniques; cependant, sur deux dédicaces offertes à Jupiter, le dédicant a une double dénomination indi-

(29) «Studium Generale», IX, 1962, p. 336-351.

(30) M. Cardozo, Algumas inscrições lusitano-romanas da região de Chaves, 1948, $.^{\circ} 3, \rho .13$ - F. Russell Cortez, Lápidas do Museu de Chaves, «Viriatis», I, 1957, n. ${ }^{\circ} \mathbf{1}$, p. 100.

(31) $E E$, IX, $277=I L E R$, 5594. P. LE Roux, L'armée romaine et Vorganisation des provinces ibériques d'Auguste à l'invasion de 409, Paris, 1982, p. 224 et 336.

(32) EE, VIII, 128. Cette inscription fait partie du dossier du signe 3. Plusieurs travaux récents ont repris la question: G. Pereira Menaut, Caelo Cadroiolonis $f$. Cilenus 3 Berisamo et al.: centuria or Castellum, a discussion, «Hispania Antiqua», VIII, 1978 (1982), p. 271-280. P. LE Roux et A. Tranoy, 3, le mot et la chose. Contribution au débat historiographique, «Lucerna» (sous presse).

(33) F. M. Alves, «Revista de Arqueologia», 3, 1936, p. 41-42.

(34) Inédit.

(35) Le RouX-Trànoy, n. ${ }^{\circ}$ 6, p. $192=A E, 1973,302$. 
gène, soit formé sur le même nom: Reb(urrius) Reburrus ${ }^{36}$ ), soit formée de deux noms différentes: Alius Reburrus ${ }^{\left({ }^{37}\right)}$.

Enfin, il est incontestable que, dans le domaine de l'onomastique, le premier résultat des contacts avec le monde romain et les indigènes fut la transposition en latin des noms indigènes. Aussi, quand nous parlons d'onomastique indigène à partir des inscriptions latines, nous utilisons déjà un matériel qui a subi une première mutation que l'on a pu définir comme un «baptême linguistique» $\left({ }^{38}\right)$.

Malgré sa richesse et sa variété, cette onomastique ne reflète pas à elle-seule toutes les composantes de la société du Tràs-os-Montes. Aussi doit-elle être complétée par d'autres données, en particulier les types de filiation.

\section{Les types de filiation (Carte II)}

Le procédé adopté pour indiquer une filiation sur une inscription est important pour apprécier les résultats des contacts entre la tradition locale et la tradition romaine. En effet, la filiation est un moyen privilégié, qui rend compte du degré réel de la progression des principes romains dans les structures familiales locales. Il y a cependant une difficulté majeure qui subsiste et que nous avons déjà soulignée: la datation délicate de la majorité des documents; pour l'instant, l'absence d'une véritable chronologie ne permet pas encore de bien saisir toute l'évolution de ces structures. Aussi est-ce surtout un tableau général que nous présentons ici, à partir de 81 cas étudiés.

Les filiations peuvent se répartir en quatre types fondamentaux $\left({ }^{39}\right)$. Le premier type correspond à une forme simple et purement indigène, où le nom d'une personne est suivi du nom différent

(36) J. Leite de Vasconcelos, Religiões da Lusitânia, III, Lisbonne, 1913, p. 223, ๆ. 2.

(37) CIL, II, 2394a = ILER, 56.

$\left.{ }^{38}\right)$ R. ÉtIENne, Les syncrétismes religieux dans la péninsule ibérique à Vépoque impériale, «Les syncrétismes dans les religions grecque, et romaine. Colloque de Strasbourg (Juin 1971)», Paris, 1973, p. 155.

( $\left.{ }^{39}\right)$ R. Étienne, G. Fabre, P. Le roux et A. Tranoy, Les dimensions sociales de la romanisation dans la péninsule ibérique des origines à la 
de son père, au génitif. Il peut s'exprimer avec une onomastique indigène: Aunia Triti (filia) $\left({ }^{40}\right)$ (Fig. 4), Cloutina Triti (filia) $\left({ }^{41}\right)$, Docia Reburri (filia) ( ${ }^{42}$ ), Docius Sanigii (filius) ( ${ }^{43}$ ), Reburrinus Bouti (filius) ( ${ }^{44}$ ) (Fig. 5), Reburrus Ari (filius) ( ${ }^{45}$ ) (Fig. 6), Reburrinus Reburri (filius )( ${ }^{46}$ ) (Fig. 7) etc... Mais l'onomastique peut être aussi à composante latine soit dans le nom du père: Alla Flavi (filia) $\left({ }^{47}\right)$, Atta Capitonis (filia) $\left({ }^{48}\right)$, soit dans celui du fils: Rufus Mosi (filius) $\left({ }^{49}\right)$, Albinus Balesini (filius) $\left({ }^{50}\right)$, soit enfin dans les noms: Fronto Capitonis (filius) ( ${ }^{51}$ ), Proculus Silonis (filius) ( ${ }^{52}$ ), Rufinus Rufi (filius) ${ }^{53}$ ). Mais la répartition entre ces groupes donne la nette prédominance aux formes indigènes seules qui représentent les $2 / 3$ de ce premier type. La seconde remarque concerne le cas où le nom du père est d'origine latine avec une descendance à nom indigène: on constate alors que ce sont essentiellement des filles qui portent ce nom indigène, ce qui semblerait indiquer que l'onomastique indigène, dans des familles où les hommes avaient adopté un nom romain, put se maintenir par l'intermédiaire des femmes.

Le deuxième type voit déjà l'introduction d'un usage romain avec la précision de la filiation marquée par la lettre $F$ ou par

fin de VEmpire, "Assimilation et résistance à la culture greco-romaine dans le monde antique. Ylème Congrès d'Études Classiques, Madrid, septembre 1972», Paris, 1976, p. 95-107; pour l'ensemble du Nord-Ouest, Tranoy, Galice romaine, p. 366-370.

(4) p M. Alves, Lápides do Museu regional de Bragança, «Memórias arqueológico-históricas do distrito de Bragança», IX, 1934, n. ${ }^{\circ}$ 38, p. 74-75, Lampaça (Bragança) (= Alves).

(41) Alves, n. ${ }^{\circ}$ 3, p. 33-34, Argozelo (Bragança).

(42) Alves, n. ${ }^{\circ}$ 19, p. 56, Donai (Bragança).

(43j CIL, II, 5070, Sanceriz (Macedo do Mato, Bragança).

(44) EE, IX, 292b, Picote (Miranda do Douro, Bragança).

(45) EE, VII, 126, Felgar (Moncorvo, Bragança).

(46) Alves, n. ${ }^{\circ} 46$, p. 84 (Deveza de Vila Nova, Bragança).

$\left.{ }^{47}\right)$ Inédit, Picote (Miranda do Douro, Bragança).

(48) Inédit, Atenor (Miranda do Douro, Bragança).

(49) J. A. Tavares, AP, XXV, 1921-1922, p. 128-133, S. Cristóvão (Moncorvo, Bragança).

(40) CIL, II, 2471, Seleiros (Chaves).

(41) Inédit, Atenor (Miranda do Douro, Bragança).

(52) CIL, II, 2510, région de Bragança. 
Fil, mais le nom du père est toujours indigène: Ama Pitili F. ( $\left.{ }^{54}\right)$, Atius Reburri F. $\left.{ }^{55}\right)$, T, Boutius Segonti F. $\left({ }^{56}\right)$ avec, pour ce cas, l'indication du prénom, Camalus Borni F. $\left.{ }^{57}\right)$, Aravius Arronis F. ${ }^{58}$ ) (Fig. 8), Deocena Careti F. $\left({ }^{59}\right)$ (Fig. 9).

Le troisième type est caractérisé par l'indication de la filiation et le nom latin du père: Flavus Festi F. $\left({ }^{60}\right)$, Sabinus Probi F. $\left({ }^{61}\right)$, Sempronius Silonis F. $\left({ }^{62}\right)$, Silvanus Severi F. $\left.{ }^{63}\right)$, Tridiae Modesti F. $\left({ }^{64}\right)$, etc... Si l'on compare les deuxième et troisième types, on remarque que, au niveau de l'onomastique des enfants, la proportion est inverse: dans le deuxième type où le père a un nom indigène, les $4 / 5$ des enfants ont aussi des noms indigènes tandis que, dans le troisième type, les $4 / 5$ des enfants ont des noms latins comme leur père. Cette caractéristique renforce la conclusion déjà notée pour l'onomastique sur l'homogénéité $\mathrm{du}$ milieu indigène et en même temps montre que, lorsque cette homogénéité est rompue, les habitudes romaines envahissent avec force l'onomastique locale. Cependant, là encore, l'exception est représentée par une femme, Tridia, fille de Modestus, qui conserve le nom indigène malgré le nom latin de son père.

Enfin, le quatrième et dernier type est constitué par la filiation par le prénom du père selon les usages classiques romains. Un seul cas a pu être noté pour cette région et encore l'inscription, mutilée, concerne-t-elle une personne étrangère au conventus puisqu'elle est originaire de Clunia $\left({ }^{65}\right)$ (Fig. 10).

(53) EE, IX, 292c, Picote (Miranda do Douro, Bragança).

${ }^{54}$ Le Roux-Tranoy, n. ${ }^{\circ} 19$, p. $211=A E$, 1973, 315, Curral de Vacas (Chaves).

(55) CIL, II, 2387, Ribeira da Pena (Vila Real).

(56) $H A E, 518$, Tresminas (Vila Pouca de Aguiar, Vila Real).

(57) CIL, II, 2484, Pastoría (Chaves).

(58) Alves, n. ${ }^{\circ}$ 39, p. 75, Rebordaos (Bragança).

(59) EE, IX, 292, Picote (Miranda do Douro, Bragança).

$\left.{ }^{60}\right)$ CIL, II, 5620, Sacoias (Bragança).

(61) CIL, II, 2495, Friães (Chaves).

(62) HAE, 1641, Moncorvo (Bragança).

(63) CIL, II, 2392, Tresminas (Vila Pouca de Aguiar, Vila Real).

(64) J. Leite de Vasconcelos, dans ^4P, XXIX, 1930-1931, p. 157-163.

(65) HAE, 517, Tresminas (Vila Pouca de Aguiar, Vila Real). 
Cette étude des filiations, conjuguée avec l'onomastique, atteste de l'importance de la tradition locale, dans un milieu indigène qui est resté fondamentalement rural.

Tableau récapitulatif

\begin{tabular}{|c|l|c|}
\hline Types & \multicolumn{1}{|c|}{ Chiffres } & Pourcentage \\
\hline I & 37 (dont 24 indigènes seuls) & $46 \%$ \\
\hline II & 33 (dont 27 avec fils à nom indigène) & $41 \%$ \\
\hline III & 10 (dont 8 avec fils à nom latin) & $12 \%$ \\
\hline IV & 1 & $1 \%$ \\
\hline
\end{tabular}

Il est enfin un dernier aspect de cette épigraphie transmontanaise que nous voudrions souligner, celui du rôle des décors dans les stèles funéraires.

Les décors funéraires: le «style de Picote» (Carte III)

Une cartographie des principaux décors funéraires du Trás-os-Montes, classés en fonction des symboles ou des représentations: astres, décor végétal, triscèles, animaux, etc..., permet de distinguer un très important foyer dans la région de Miranda do Douro, plus particulièrement autour du village de Picote, avec une diffusion des décors vers le Nord du Portugal (région de Bragança) et, en Espagne, vers Zamora et la Tierra de Aliste. Aussi pensons-nous pouvoir attribuer à cette catégorie de documents le nom de «style de Picote».

Comment se définissent les caractéristiques de ce style d'iconographie funéraire ${ }^{66}$ ) ? Faite en marbre de la région de Miranda ou en granit, la stèle-type comporte plusieurs registres (Fig. 11).

(66) Pour la présentation générale, J. Leite de Vasconcelos, Religiões da Lusitânia, Lisbonne, 1913, III, p. 406-443; voir aussi, Tranoy, Galice Romaine, p. 340-359.

Conimbriga, 23 (1984), 19-41 
La partie supérieure est arrondie et décorée par une roue à rayons courbes, inspirée du triscóle celtique; ce motif se détache et forme lui-même une sorte de stèle à sommet circulaire à l'intérieur du cadre du monument. Il est entouré de petits disques, complétés parfois par un décor végétal en feuilles de lierre; les angles sont soulignés par des équerres. Le champ épigraphique forme le deuxième panneau sous lequel est sculpté un motif animalier: taureau, sanglier, cerf, biche..., pouvant être en rapport avec le ou les défunts de la stèle. Enfin, le dernier registre est constitué d'une série d'arcatures dont la forme en «fer à cheval» n'est pas sans parenté avec ce que sera l'arc visigotique quelques siècles plus tard $\left({ }^{67}\right)$. Ces stèles sont assez souvent géminées comme à Cercio ${ }^{68}$ ) ou à Aldeia Nova $\left({ }^{69}\right)$ (Fig. 12).

L'intérêt de ces monuments est de nous présenter trois niveaux différents d'interprétation et d'acculturation. En effet, la fréquence du décor animalier avec le thème du sanglier ou du porc est à rapprocher des grandes sculptures protohistoriques des «Berrões», dont l'un des foyers essentiels fut le Tràs-os-Montes $\left({ }^{70}\right)$; l'insertion de ces animaux dans le décor funéraire à l'époque romaine est le témoignage de la survivance des anciens rites religieux indigènes. En revanche, il est possible d'attribuer au milieu celtique l'importance prise par les astres et les rouelles solaires sur les monuments funéraires de la région de Picote; on retrouve là un thème décoratif fréquent dans les castros. Enfin, la conception d'ensemble du monument, une grande stèle avec un champ épigraphique, correspond à l'adaptation des coutumes indigènes aux modes funéraires romaines. Ces documents mettent donc en valeur l'importance du patrimoine culturel des peuples établis à l'Est du Tràs-os-Montes, probablement les Zoelae. Une stèle funéraire de León confirme l'attachement de ce peuple

(67) J. Fontaine, L'art préroman hispanique (La nuit des temps, 38), Paris, 1973, p 127-128.

$\mathrm{H}$ Inédit.

H EE, VIII, 129-130.

(70) J. R. Dos Santos Junior, A cultura dos berrões no Nordeste de Portugal, TAE, XXIII, 1975, p. 353-515, complété dans TAE, XXIII, 1977, p. 5-18 et p. 333-345.

Conimbriga, 23 (1984), 19-41 
à ses traditions. En effet, le peuple des Zoelae est représenté à León par un autel dédie à T. Montanius Fronto, custos armorum, qui mentionne son origo, dois Z(o)elae $\left.{ }^{71}\right)$. En revanche, Vorigo ne figure pas sur la stèle de Lucretius Proculus, lui-aussi custos armorum à León $\left({ }^{72}\right)$, mais le décor de cette stèle est du même type que le décor des stèles de Picote et est unique dans les stèles de León (Fig. 13). N'est-ce pas là un indice révélateur de l'origine de Lucretius Proculus, mort à León avec sa femme et son fils, mais qui venait probablement du Tràs-os-Montes, évoqué sur la stèle par le décor funéraire où la représentation des animaux est étroitement en rapport avec les personnes mentionées? Ces exemples confirment bien la nécessité de l'étude des supports épigraphiques dont la richesse peut apporter un éclairage à des textes souvent très laconiques.

\section{Conclusion}

Pour conclure cette présentation d'ensemble de l'épigraphie $\mathrm{du}$ Nord du Portugal, nous voudrions insister sur plusieurs points.

En premier lieu, ce bilan met en évidence la richesse épigraphique de cette région et la grande variété des inscriptions permet de pallier en partie l'absence de documents littéraires, bien que les obstacles soient très nombreux. C'est en multipliant les comparaisons entre les documents que l'on pourra peut-être retrouver à la fois les traces d'ateliers locaux et des critères paléographiques qui, pour l'instant, restent encore très incertains. Ce n'est qu'à ces conditions que l'on pourra proposer des solutions aux problèmes chronologiques.

Il est donc nécessaire d'encourager tous les efforts de prospection et d'enquêtes locales qui, seules, permettront d'augmenter le nombre des inscriptions recensées dans cette région.

Mais ces recherches ne seront d'un réel intérêt que si elles débouchent sur une publication du matériel découvert. C'est à

(71) CIL, II, 5684 .

(72) CIL, II 2668. 
partir de ces travaux que pourront alors se dégager les caractères originaux de cette épigraphie qui reste la base de toute étude régionale de l'histoire antique du Nord du Portugal. Elle constitue en ce sens un modèle pour la compréhension d'une histoire où les structures politiques et administratives n'interviennent que comme un élément à côté des données matérielles et des mentalités.

\section{Addenda:}

Depuis la rédaction de cet article, de nouvelles inscriptions ont été découvertes et ont donné lieu à des publications.

Il faut citer tout d'abord la statue de guerrier avec inscription sur le bouclier, provenant de Refojos de Basto: A. Coelho F. DA SILVA, NOVOS dados sobre a organização social castreja, "Portugalia», II-III, 1981-2, p. 83-94, où l'auteur présent en même temps de nouvelles inscriptions de la région de Chaves, en particulier la borne honorifique découverte en 1980 dans le Tâmega, texte original de la «Padrão dos Povos». La statue du guerrier est aussi étudiée par C. A. Ferreira De AlmeIDA, Nova estátua de guerreiro galaico-minhoto (Refojos de Basto), «Arqueologia«, 3, 1981, p. 111-116. cet article a été à l'origine d'une discussion: A. Coelho F. DA SILvA, Uma carta a propósito do guerreiro de Refojos de Basto, «Arqueologia», 5, 1982, p. 80-82 et C. A. Ferreira de Almeida, Resposta, «Arqueologia», 5, 1982, p. 82-84. D'autre part,

l'épigraphie sert de support à l'article de A. Coelno F. da Silva, Organizações gentilicias entre Leça et Ave, «Portugalia», I, 1980, p. 79-90.

Les régions de Chaves et de Braga ont fourni des dédicaces à Jupiter et des inscriptions funéraires: C. A. Ferreira DE Almeida, Nova e importante ara a Júpiter (Fiães, Valpaços), «Arqueologia», 5, 1982, p. 69-70; P. LE Roux et A. Tranoy, Nouvelles inscriptions du Nord du Portugal, «Minia», 6, 1982, p. 31-37. Une stèle funéraire de Favaios (Alijó) a été étudiée par C. A. Brochado de Almeida et M. Alves Plácido, a estela funerária romana da Quinta de S. Jorge (F av aios-Alijó), «Arqueologia», 7, 
1983, p. 48-51. De son côté, la nouvelle revue «Brigantia» publie quelques inscriptions funéraires de la région de Bragance: B. Afonso et J. M. MoraIs, Brigantia, I ${ }^{1}, 1981$, p. 82 et B. Afonso, Brigantina, $\mathrm{I}^{2}$, 1981, p. 82 et B. Afonso, Brigantina, $\mathrm{I}^{2}, 1981$, p. 148 et 150 .

Enfin, une importante collection épigraphique inédite va être présentée par L. A. dos Santos, P. LE Roux et A. Tranoy, Inscrições romanas do Museu Pio XII em Braga, «Bracara Augusta» (sous presse).

A travers toutes ces publications, on peut constater la vitalité de la recherche épigraphique dans le Nord du Portugal.

Abréviations

$\begin{array}{ll}A E & \text { Année Épigraphique. } \\ A P & \text { O Arqueólogo Português. } \\ C A N & \text { Congreso Nacional de Arqueología (Espagne). } \\ C I L & \text { Corpus Inscriptionum Latinarum. } \\ C N A \text { Congresso Nacional de Arqueologia (Portugal). } \\ E E \quad \text { Ephemeris Epigraphica. } \\ \text { HAE } \quad \text { Hispania Antiqua Epigraphica. } \\ I L E R \text { Inscripciones Latinas de la España Romana. } \\ M C V \quad \text { Mélanges de la Casa de Velázquez. } \\ R G \quad \text { Revista de Guimarães. } \\ T A E & \text { Trabalhos de Antropologia e Etnologia. }\end{array}$




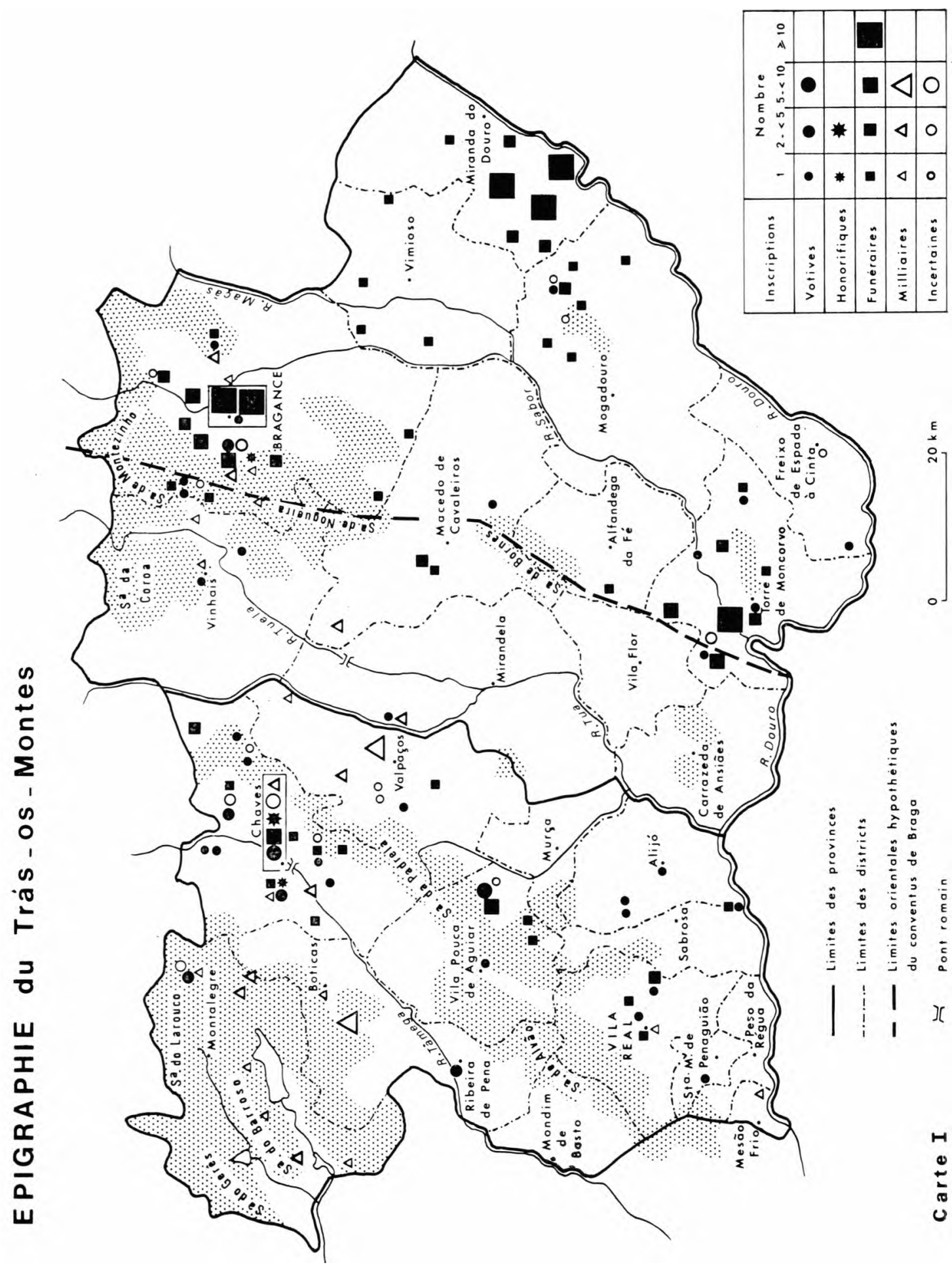




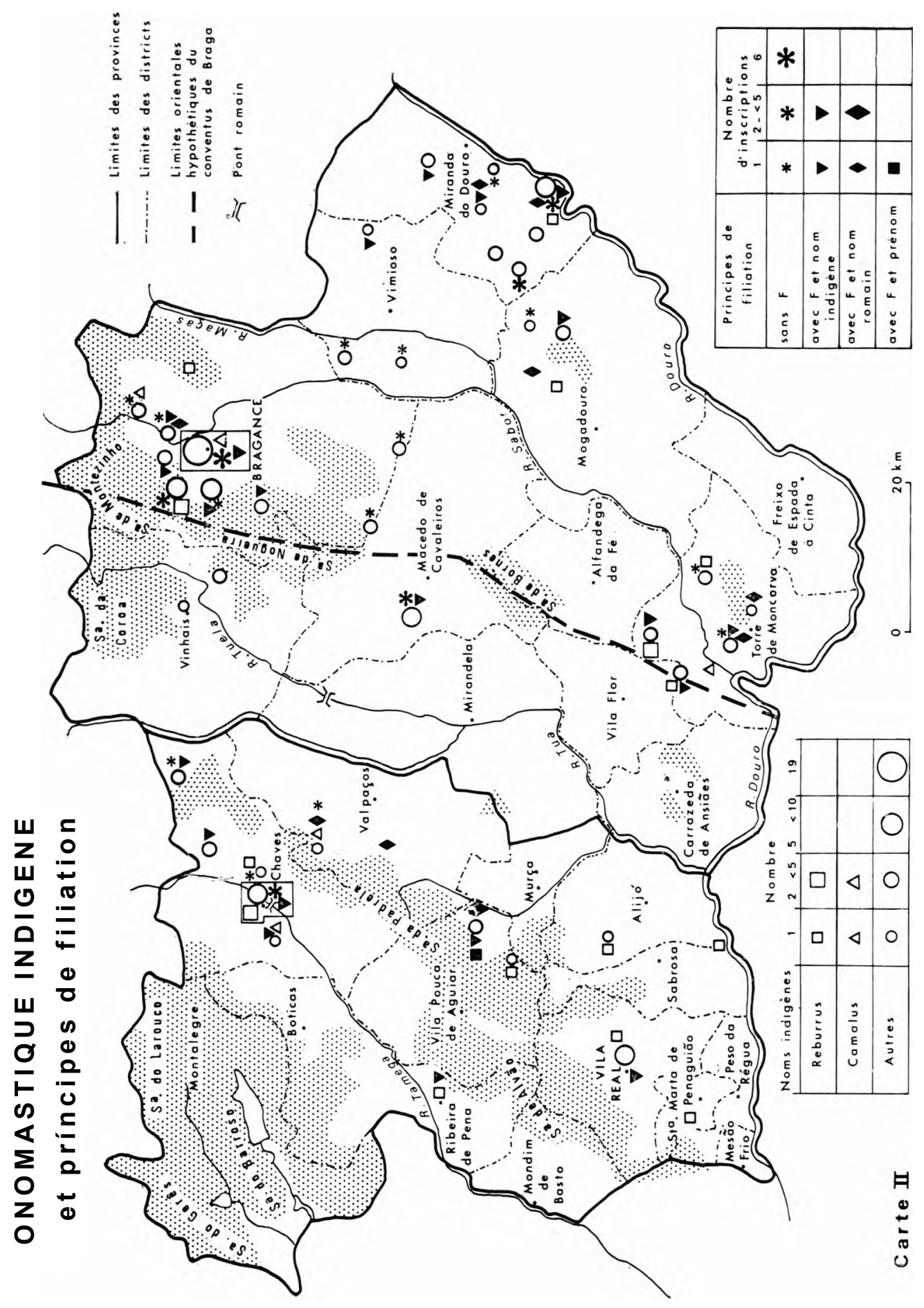








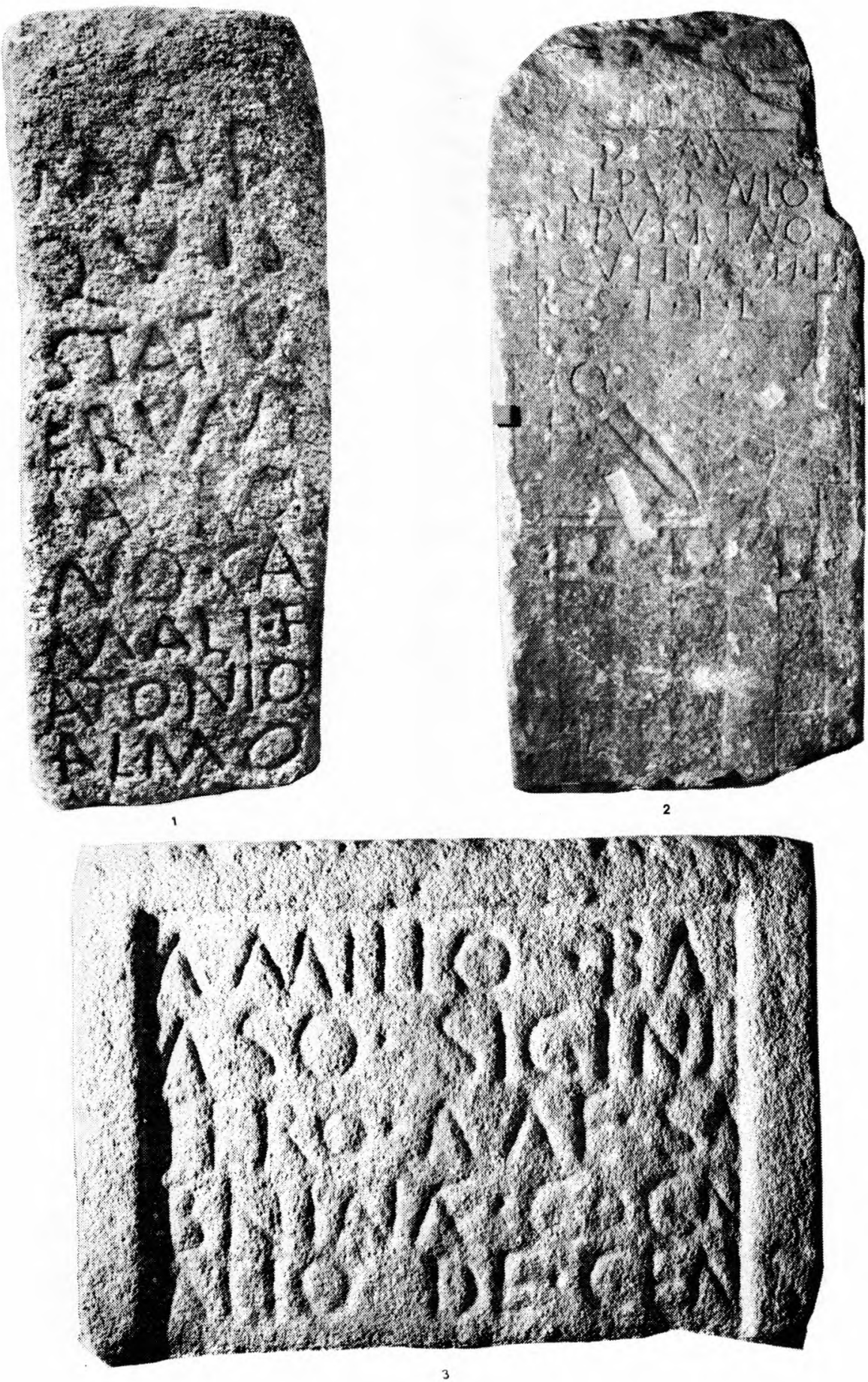

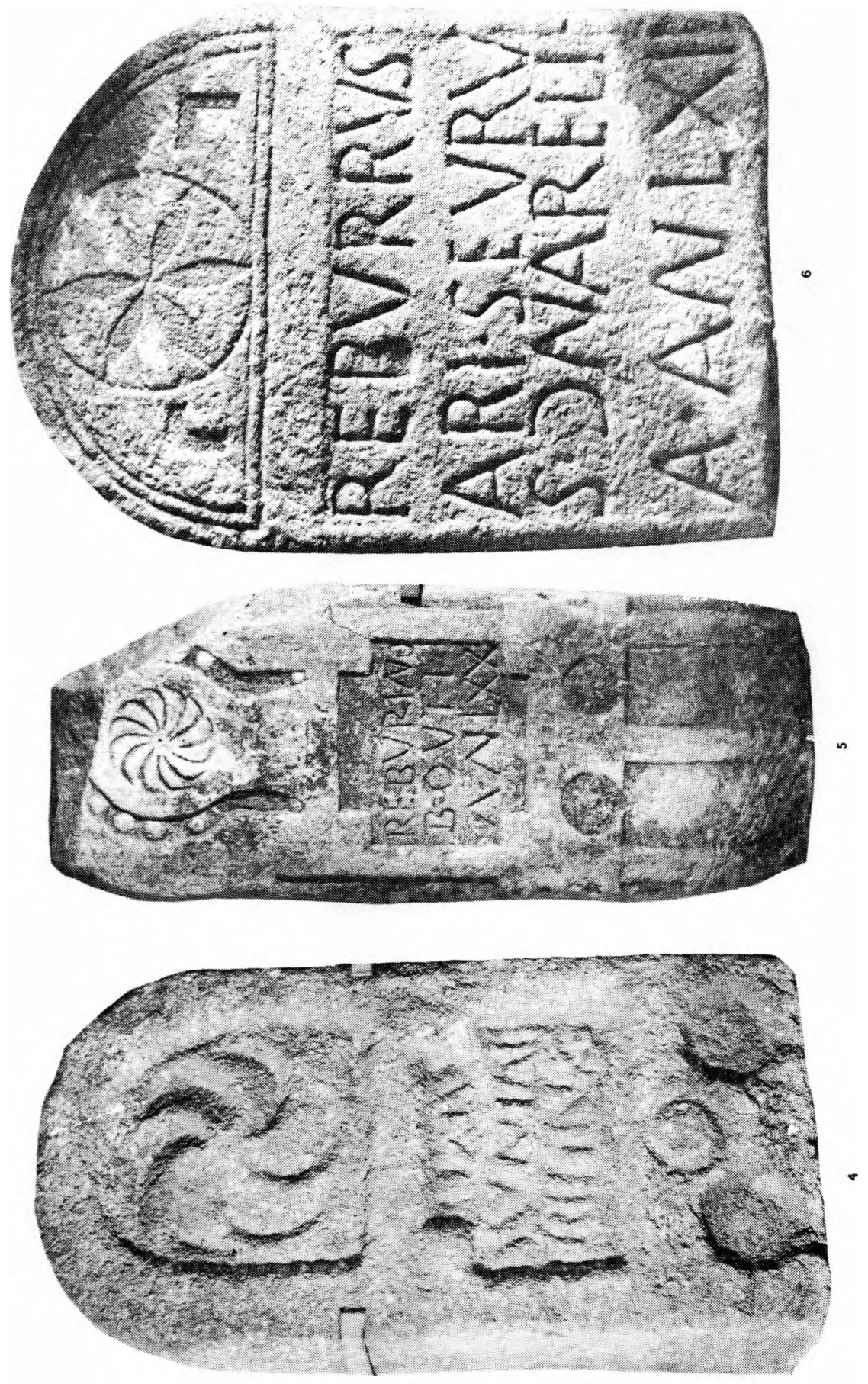

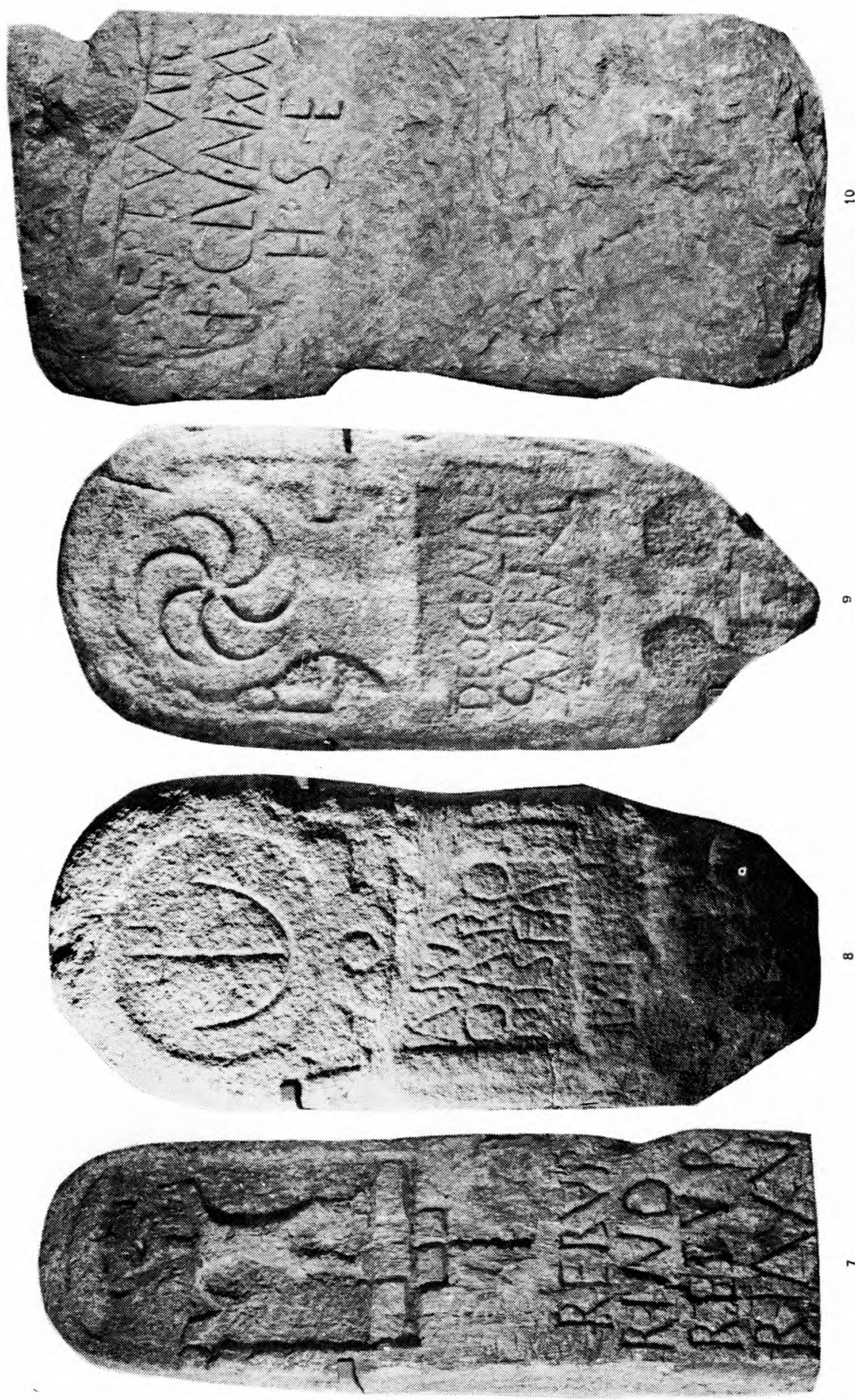

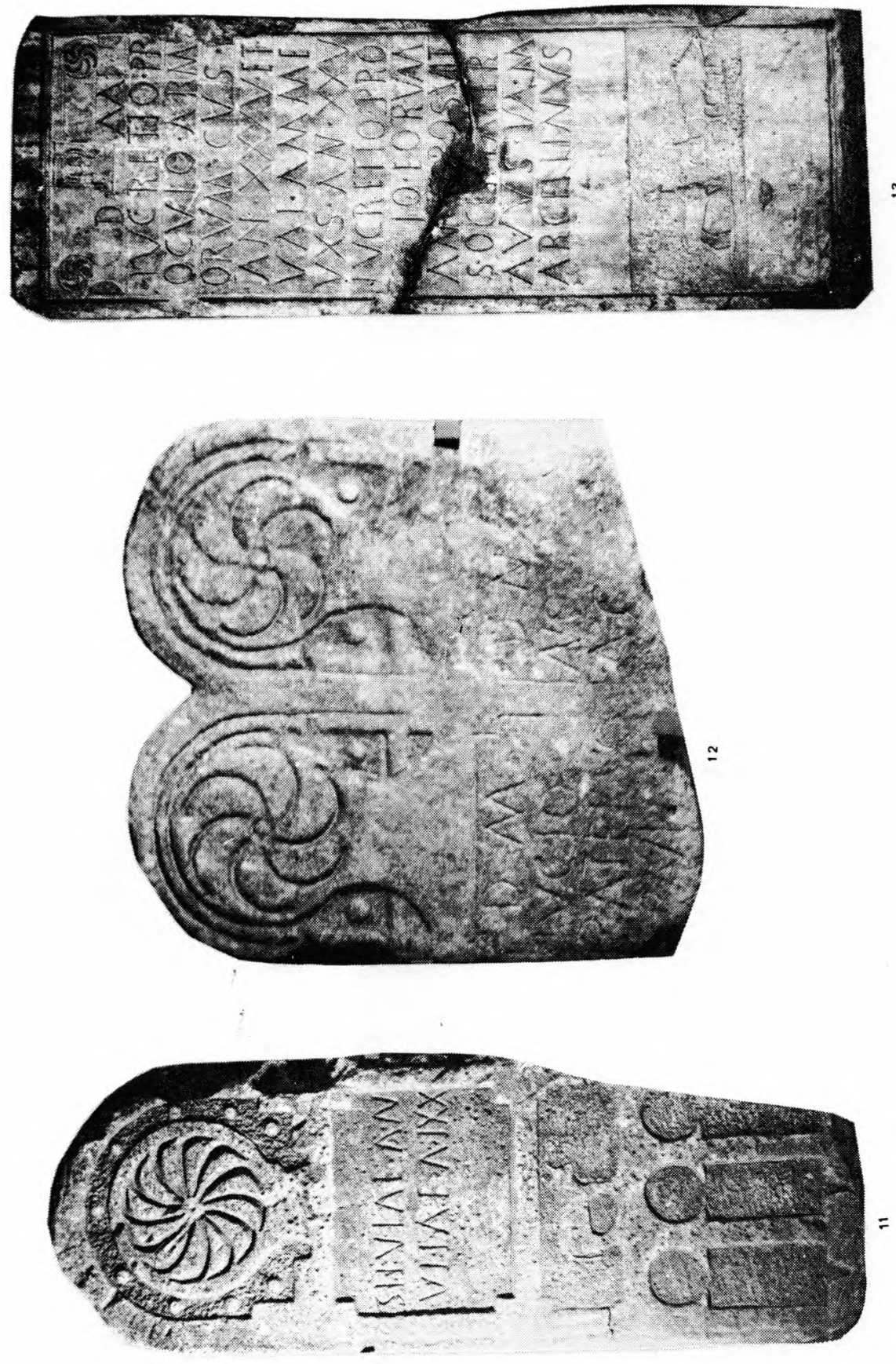